\title{
Análisis de la Carta de la ONU a la luz de los postulados del texto "Zum ewigen Frieden"* de Immanuel Kant**. El objetivo de la paz y el mecanismo de la guerra
}

\author{
Analysis of the UN Charter in Light of the Postulates \\ of Immanuel Kant's "Zum ewigen Frieden." The \\ Objective of Peace and the Mechanisms of War
}
Análise da Carta da ONU à luz dos postulados do texto "Zum ewigen Frieden" de Immanuel Kant. 0 objetivo da paz e o mecanismo da guerra

Joe Nayib Campos Salazar ${ }^{* * *}$

Fecha de recepción: 9 de febrero de 2019
Fecha de aprobación: 13 de marzo de 2019

Doi: $h t t p: / / d x$. doi.org/10.12804/revistas.urosario.edu.co/anidip/a.8500

Para citar: Campos Salazar, J.N. (2019). Análisis de la Carta de la ONU a la luz de los postulados del texto "Zum ewigen Frieden" de Immanuel Kant El objetivo de la paz y el mecanismo de la guerra. ANIDIP (7), 116-140. Doi: http://dx.doi.org//10.12804/revistas.

urosario.edu.co/anidip/a.8500

\section{Resumen}

En el presente ensayo se procede a realizar un análisis de la Carta de las Naciones Unidas, específicamente en relación con el objetivo de mantener la paz y, cómo este mismo documento regula los conflictos que pueden poner en riesgo tal objetivo. El análisis propuesto intentará fusionar las ideas emanadas del texto político de Kant,

* Traducido al español como «Sobre la paz perpetua». Una traducción más precisa sería "Hacia la paz eterna o perpetua".

** Un agradecimiento a David A. Sánchez Elizondo y Joroge Umaña Varģas por sus valiosos comentarios, esenciales para la finalización del ensayo, a las sugerencias de Ariana Macaya Lizano, que fueron precursoras para el desarrollo de este escrito, y al jurado del Certamen de Estudios Críticos sobre la Justicia por sus atinentes recomendaciones para el mejoramiento del ensayo.

*** Bachiller en Derecho y egresado de la licenciatura en Derecho de la Universidad de Costa Rica. Actualmente cursa la maestría profesional en Derechos Humanos de la Universidad Estatal a Distancia y el bachillerato en Filosofía de la Universidad de Costa Rica. Disciplina de Filosofía.

Correo electrónico: joenayib@ǵmail.com 
el cual establece pautas de cómo debe ser un tratado dirigido al establecimiento de la paz perpetua.

En la primera sección se analiza el Capitulo I: Propósitos y principios en concordancia con el Capitulo VI: Arreglo pacífico de controversias y con las medidas que se separan del concepto propio de la paz, las cuales se encuentran en el Capitulo VII: Acción en caso de amenazas a la paz, quebrantamientos de la paz o actos de agresión. En la segunda sección se describe el texto kantiano Zum ewigen Frieden y, a la vez, se analizan los artículos del modelo de tratado que se establece.

En la última sección se realiza un análisis correlacional entre la Carta de la Naciones Unidas y el modelo kantiano determinando la existencia de una antinomia normativa que implica la imposibilidad material en el cumplimiento del objetivo de mantener la paz.

Palabras clave: paz, Carta de las Naciones Unidas, paz perpetua, guerra, Kant.

\section{Abstract}

This essay analyses the objective of maintaining peace, contained in the Charter of the United Nations, and how it regulates the conflicts that may put it at risk. The proposed analysis will attempt to merge the ideas emanating from Kant's political text, which sets guidelines for how a treaty aimed at achieving perpetual peace should be. The first section analyzes Chapter I (Purposes and Principles) of the UN Charter, in accordance with Chapter VI (Pacific Settlement of Disputes) and with the measures found in Chapter VII (Action with Respect to Threats to the Peace, Breaches of the Peace and Acts of Aggression), which present a conflict when compared with the notion of peace. The second section describes the Kantian text Zum ewigen Frieden and explains the articles of the model treaty. The last section consists of a comparative analysis of the un Charter and the Kantian model. Finally, the analysis led to conclude there is a normative antinomy that results in a material impossibility to fulfill the objective of maintaining peace.

Key words: peace, Charter of the United Nations, perpetual peace, war, Kant.

\section{Resumo}

No presente ensaio procede-se a realizar uma análise da Carta das Nações Unidas, especificamente em relação com o objetivo de manter a paz e, como este mesmo documento regula os conflitos que podem pôr em risco tal objetivo. A análise proposta tentará fusionar as ideias emanadas do texto político de Kant, o qual estabelece pautas de como deve ser um tratado dirigido ao estabelecimento da paz perpetua. Na primeira seção se analisa o Capitulo I: Propósitos e princípios em concordância com o Capitulo VI: Acordo pacífico de controvérsias e com as medidas que se separam 
do conceito próprio da paz, as quais se encontram no Capítulo VII: Ação em caso de ameaças à paz, quebrantamentos da paz ou atos de agressão.

Na segunda seção se descreve o texto kantiano Zum ewigen Frieden e, ao mesmo tempo, se analisam os artigos do modelo de tratado que se estabelece.

$\mathrm{Na}$ última seção se realiza uma análise de correlação entre a Carta das Nações Unidas e o modelo kantiano determinando a existência de uma antinomia normativa que implica a impossibilidade material no cumprimento do objetivo de manter a paz.

Palavras-chave: paz, Carta das Nações Unidas, paz perpetua, guerra, Kant.

\section{Introducción}

El presente ensayo analiza la Carta de las Naciones Unidas (en adelante también denominada "La Carta") desde el texto Zum ewigen Frieden del autor Immanuel Kant. Principalmente, este análisis versará sobre el concepto de la paz como objetivo primario de la creación de la Naciones Unidas (ONU) y cómo se desarrolla en los diversos capítulos de dicha Carta a la luz de los postulados kantianos del texto indicado.

El objeto de este ensayo es analizar el propósito central consagrado en la Carta de las Naciones Unidas, junto con los demás artículos que se refieren respectivamente a la paz y a su antónimo político (la guerra), pues se revisa la posible concordancia presente entre ellas, desde la perspectiva kantiana transmitida en el texto supra mencionado. A su vez, este ensayo vislumbrará la posibilidad que, desde los postulados del texto kantiano, la Carta de las Naciones Unidas resulta un tratado que podría no cumplir su objetivo, o sí. Dicha afirmación será tratada como el problema al cual se plantea ofrecerle respuesta.

El estudio de este tema, en conjunto con el abordaje desde la perspectiva kantiana, presenta gran relevancia en la actualidad, pues pretende analizar el funcionamiento de las Naciones Unidas en cumplimiento con su objetivo primario y las posibles deficiencias de los mecanismos empleados para tal fin, aproximadamente a los 74 años de la promulgación de su carta constitutiva.

Para el adecuado desarrollo del presente ensayo se partirá de la hipótesis que, asumiendo una posición kantiana, se logra evidenciar una contradicción en el documento de la Carta de la Naciones Unidas. Así, desde una perspectiva teleológica, se puede identificar la imposibilidad material de las Naciones Unidas para cumplir su fin, puesto que desde su propio origen se deriva una obstaculización formal.

En otras palabras, el motivo por el cual las Naciones Unidas no han podido cumplir su objetivo primario se debe a que se crearon pautas antagónicas con el 
concepto de paz, es decir, "La Carta" incurre en una antinomia normativa, esto se ha denotado en el plano ontológico y empírico de dicha entidad.

\section{Carta de las Naciones Unidas}

La Carta de las Naciones Unidas es el cuerpo normativo que crea y fundamenta la Organización de las Naciones Unidas (ONU). Fue el resultado de los conflictos bélicos y políticos de orden global que acontecieron durante la primera mitad del siglo XX.

Como antecedente histórico inmediato cabe mencionar la Liga de Naciones creada en 1919, la cual presenta objetivos similares a los que adoptó la Carta de las Naciones Unidas en su cuerpo constitutivo; sin embargo, por la falta de apoyo de ciertos Estados influyentes en el entorno de entonces no resultó efectiva en su funcionamiento. Debido a la inefectividad de esta organización internacional, y luego de revisar los atroces resultados posteriores a la Segunda Guerra Mundial, muchos grupos de influencia política y económica global analizaron la necesidad de crear otro organismo diferente a la Liga de Naciones, que intentara responder en forma adecuada a la realidad de ese contexto internacional.

La constitución de dicho cuerpo normativo, según Santa Cruz (1995): "concibe un orden mundial y coloca al ser humano como centro principal de su interés y de su acción, en su calidad de individuo, de ciudadano, y de miembro de una raza regida por principios de igualdad, justicia y solidaridad" (p. 21). Esto responde a la presión de ciertos movimientos políticos derivados de las guerras, los cuales se apegaron a los derechos naturales y a las libertades humanas que desde 1948 se denominaron "derechos humanos", a partir de la Declaración Universal de Derechos Humanos; a la necesidad de la eliminación de los conflictos en pro de respetar y preservar estos derechos humanos, y a la búsqueda de mecanismos que eviten la repetición de los acontecimientos de la primera mitad del siglo XX.

\subsection{Propósitos y principios}

La Carta de las Naciones Unidas, en el artículo primero que corresponde a los propósitos y principios, establece lo siguiente:

Los Propósitos de las Naciones Unidas son: 1. Mantener la paz y la seguridad internacionales, y con tal fin: tomar medidas colectivas eficaces para prevenir y eliminar amenazas a la paz, y para suprimir actos de agresión u otros quebrantamientos de la paz; y lograr por medios pacíficos, y de conformidad con los principios de la justicia y del derecho internacional, el ajuste o arreglo de controversias o 
situaciones internacionales susceptibles de conducir a quebrantamientos de la paz (Naciones Unidas, 2015, p. 4).

El artículo primero indica el telos de "La Carta" y, en general, el funcionamiento de la Organización de las Naciones Unidas; por lo tanto, resulta natural suponer que el resto de los artículos, desde una interpretación sistemática normativa, deberían responder a esta pauta. Así mismo, el artículo primero amplía sus propósitos, pues agrega la idea de fomentar las relaciones amistosas, lograr la cooperación internacional y ser el encargado de armonizar los esfuerzos de las naciones.

Es importante señalar cómo el artículo primero indica que con el fin de mantener la paz se podrán "tomar medidas colectivas eficaces para prevenir y eliminar amenazas" a esta; por ello, en el mismo documento se establecen dos capítulos orientados a lograr tal fin. El primero, desde la paz misma y el segundo desde fuera de esta, es decir, el primero se crea en un ambiente donde no concurran hostilidades y se apela a medios diplomáticos, mientras en el segundo escenario se acude a medidas represivas y hostiles; incluso es válida la utilización de fuerzas castrenses. Desde esta perspectiva, se empieza a vislumbrar una tensión normativa al interior de la Carta de la Naciones Unidas.

El concepto de la paz se vuelve condición necesaria para lograr los propósitos mencionados en el párrafo anterior. En otras palabras, para el establecimiento de relaciones amistosas se debe presentar un ambiente de paz anticipado; pues de lo contrario, un vínculo amistoso no sería viable entre las partes, sino más bien uno de interés, no de verdadera colaboración entre los Estados. Esto mismo sucede con la cooperación internacional, puesto que la existencia de un estado de guerra conlleva a la individualización estatal, contrario sensu, el estado de paz es necesario para la solidaridad estatal. Y la armonía entre las partes es el resultado de la concurrencia de un ambiente pacífico, en el cual converjan los otros dos objetivos. Por tal motivo, la paz es el meollo de los propósitos de las Naciones Unidas, los otros objetivos resultan accesorios o colaterales a este.

Así mismo, es interesante comprender cómo en la Carta de las Naciones Unidas se presenta una visión alimentada por los hechos acaecidos en el contexto de postguerra, y se enfatiza en el hecho de que los Estados se encuentran en paz: primero, se evidencia cómo se utiliza el verbo "mantener", es decir, ya se encuentra dado y no es una acción que se deba lograr, conseguir o implementar. Segundo, se indica que se deben "eliminar" amenazas o quebrantamientos a la paz, esto reafirma la condición de esta como un concepto dado. Tercero, en el capítulo VI, los arreglos son para evitar el "peligro a la paz". Este mismo fenómeno se presenta de manera 
reiterada en dicho documento, por lo tanto, se infiere que quienes lo idearon determinaron la existencia de un ambiente de paz, el cual era necesario proteger y no un ambiente en donde se debía lograr.

Esta precisión de términos es necesaria, ya que la Carta de las Naciones Unidas está redactada en función de "proteger", lo cual supone su existencia en un escenario de paz. No obstante, se debate si hay un escenario de paz logrado y no que este todavía debería ser una meta propiamente. En ese sentido, las medidas a seguir para proteger la paz variarían según si se comprende esta como un concepto logrado o por lograr, pero, como se ha indicado, "La Carta" sigue la primera postura.

\subsection{Arreglo pacífico de controversias}

La paz es un concepto dado en la Carta de las Naciones Unidas, y, por lo tanto, debe dársele la protección que se deriva en el momento de ratificarse ese documento jurídico internacional. Por dicho motivo, esta indica lo siguiente en su prólogo:

[Y] con tales finalidades, a practicar la tolerancia y a convivir en paz como buenos vecinos, a unir nuestras fuerzas para el mantenimiento de la paz y la seguridad internacionales, a asegurar, mediante la aceptación de principios y la adopción de métodos (Naciones Unidas, 2015, p.3).

Desde el prólogo, se establece que se adoptarán métodos, medidas y principios para mantener la paz, lo cual se reafirma en el artículo 1 de la Carta de las Naciones Unidas indicado supra, pues define que este se logrará "por medios pacíficos, y de conformidad con los principios de la justicia y del derecho internacional, el ajuste o el arreglo de controversias" (Naciones Unidas, 2015, p. 4).

El término controversia debe comprenderse como lo indicó la Corte Permanente de Justicia Internacional, al afirmar que:

[C]ontroversia era "un desacuerdo sobre un punto de derecho o de hecho, una contradicción, una oposición de tesis jurídicas o de intereses entre dos personas". Esta definición, adoptada en sus inicios, ha sido utilizada por la jurisprudencia que ha desarrollado el Tribunal Internacional de Justicia (TIJ) hasta nuestros días (Sánchez Patrón, 2013, p. 129). ${ }^{1}$

1 El Tribunal Permanente de Justicia Internacional manifestó esta frase en "Asunto de las concesiones Mavrommatis en Palestina, sentencia de 30 de agosto de 1924, CPJI, Serie A, no 2, (p.11)”. 
Evidentemente, el contenido del concepto empleado por la Corte presenta una gran abstracción, esto responde a la necesidad de determinación casuística para tener competencia de conocer diversos escenarios en donde concurran conflictos.

Por otra parte, si bien el artículo primero indica cuáles son las pautas para el mantenimiento de su fin, puede ser insuficiente para el resguardo efectivo de la paz. Por tal motivo, en la misma Carta se establece el Capítulo VI: Arreglo pacífico de controversias, contenido en los artículos 33 al 38.

Según el artículo 33 de la Carta, el arreglo pacífico de controversias busca una "solución, ante todo, mediante la negociación, la investigación, la mediación, la conciliación, el arbitraje, el arreglo judicial, el recurso a organismos o acuerdos regionales u otros medios pacíficos de su elección" (Naciones Unidas, 2015, p. 24), lo anterior en virtud de mantener la paz y evitar cualquier peligro a esta. Del mismo modo, en el artículo 34 de la Carta, se autoriza al Consejo de Seguridad a "toda situación susceptible de conducir a fricción internacional o dar origen a una controversia, a fin de determinar si la prolongación de tal controversia o situación puede poner en peligro el mantenimiento de la paz y la seguridad internacionales" (Naciones Unidas, 2015, pp. 24-25).

En el derecho internacional, los medios alternos de resolución de conflictos suelen ser utilizados por los Estados, pues son la forma de no sufrir una sanción, sino que permiten llegar a un acuerdo donde los intereses de ambas partes sean escuchados y ponderados para obtener un resultado. Por este motivo "el arbitraje internacional goza de buena salud, tanto en la práctica contenciosa como en la convencional; al mismo tiempo, nos encontramos también con que en este procedimiento de arreglo se siguen consolidando aspectos o dimensiones" (Sáenz de Santa María, 2013, p. 79) que buscan responder a los retos contemporáneos.

En el ámbito práctico, “destacan lo que podríamos llamar los 'tres [í]conos' del arbitraje internacional en el momento presente: la Corte Permanente de Arbitraje (CPA) revitalizada, la importancia del arbitraje del anexo VII de la Convención de las Naciones Unidas sobre el Derecho del Mar (CNUDM) y los arbitrajes de inversiones" (Sáenz de Santa María, 2013, p. 80). Por lo tanto, existen diversas entidades que pretenden resolver pacíficamente los problemas de los Estados, así como de otras entidades con personalidad jurídica internacional.

Además, con el fundamento de que se debe "utilizar la negociación política en sus múltiples modalidades como forma pacífica de gestión de crisis" (Sanz Caballero, 2008, p.61), aparece la figura de la negociación preventiva, la cual "evita que surjan las controversias o bien impide que las ya existentes se intensifiquen $\mathrm{y}$ aboquen en un enfrentamiento armado. Se trata del mecanismo [...] que, 
cuando tiene éxito, conjura el peligro del conflicto antes de que éste aparezca" (Sanz Caballero, 2008, p. 61).

En otras palabras, los arreglos pacíficos pueden surgir desde que exista una posibilidad de amenaza al conflicto, pues se busca que no se llegue a un escenario de hostilidades diplomáticas y, mucho menos, bélicas. Por lo tanto, los Estados Miembros, según el artículo 35 de La Carta, podrán llevar estas situaciones ante el Consejo de Seguridad para que este intervenga y dé las consideraciones pertinentes de acuerdo con las disposiciones normativas supra planteadas, así como "recomendar los procedimientos o métodos de ajuste que sean apropiados" (Naciones Unidas, 2015, p. 26) o "los términos de arreglo que considere apropiados" (Naciones Unidas, 2015, p. 27), conforme a los artículos 36 y 37 de La Carta, respectivamente.

Las Naciones Unidas, en pro de lograr su objetivo primario de un ambiente internacional de paz y según el mismo artículo 35, pueden aceptar controversias de Estados no Miembros, siempre que estos hayan aceptado previamente La Carta, al menos en lo relativo a las obligaciones de arreglo pacífico de controversias, es decir, el Capítulo VI. De igual forma, según el artículo 36, las “controversias de orden jurídico, por regla general, deben ser sometidas por las partes a la Corte Internacional de Justicia” (Naciones Unidas, 2015, p. 26).

En síntesis, se pueden indicar que los arreglos pacíficos se pueden desglosar de la siguiente manera:

1. Negociación, investigación, mediación, conciliación, arbitraje y arreglo judicial.

2. Se pueden presentar recursos a organismos internacionales o establecer acuerdos regionales u otros medios pacíficos de su elección.

3. El Consejo de Seguridad se encargará de la continuación del desacuerdo de las partes, si los medios indicados no logran disolver la disputa.

4. El Consejo de Seguridad siempre podrá dar recomendaciones a las partes de cómo proceder o resolver el desacuerdo.

5. El Consejo de Seguridad podrá interferir cuando estime que dicha disputa podrá poner en peligro el estado de paz.

Resulta evidente que estos conflictos parten del hecho de la comunicación entre las partes y, en caso de que las partes no puedan tener una óptima comunicación, convocar a un tercero, tal como lo es el Consejo de Seguridad o algún otro ente mediador o de arbitraje. Lo anterior ofrece la posibilidad a los Estados y las Naciones Unidas de contar con diversos mecanismos para siempre salvaguardar el objetivo de la paz. 


\subsection{Acción en caso de amenazas a la paz, quebrantamientos de la paz 0 actos de agresión}

En la Carta de las Naciones Unidas se comprueba la contradicción desde su prólogo (supra citado), pues se establece que en función de lograr sus fines los Estados se comprometen "a unir nuestras fuerzas para el mantenimiento de la paz y la seguridad internacionales, a asegurar, mediante la aceptación de principios y la adopción de métodos, que no se usará; la fuerza armada sino en servicio del interés común" (Naciones Unidas, 2015, p. 3).

Esta mención en el prólogo es de interés, pues se refiere al mantenimiento y la legitimación de actuar de las fuerzas armadas bajo ciertos escenarios. Si bien se aprueba la utilización de las fuerzas armadas para el servicio del interés común, este desde una interpretación sistemática debería ser el mantenimiento de la paz, el cual se estipula como objetivo primario.

En la Carta de la Naciones Unidas se halla el Capítulo VII: Acción en caso de amenazas a la paz, quebrantamientos de la paz o actos de agresión, en el cual se desarrolla desde el artículo 39 hasta el 51.

El artículo 40 de La Carta sigue una línea de pensamiento concordante con la sección anteriormente expuesta. Igualmente, el artículo 41 establece medidas que, si bien pueden tener un gran impacto coercitivo, no utilizan el uso de la fuerza armada. En esencia, este artículo se mantiene dentro del ámbito de las medidas desde la paz misma, pero establecen la línea limítrofe entre ambos tipos.

Ahora bien, los artículos 40 (medidas provisionales) y 41 (medidas que no implican fuerza armada como la interrupción parcial o total de las relaciones económicas y las comunicaciones en su totalidad) de la Carta de las Naciones Unidas, también siguen una línea de pensamiento concordante con la sección anterior. Ahora bien, vendrían a ser los más severos al dejar de lado la diplomacia y optar por acciones coercitivas.

A partir del artículo 42 es cuando la forma de tratar los conflictos adquiere una noción diferente, ya que establece el uso de las "fuerzas aéreas, navales o terrestres, la acción que sea necesaria para mantener o restablecer la paz y la seguridad internacionales. Tal acción podrá comprender demostraciones, bloqueos y otras operaciones ejecutadas por fuerzas aéreas, navales o terrestres" (Naciones Unidas, 2015, p.29). Por otra parte, es de especial interés que estas medidas sean implementadas cuando las acciones diplomáticas o alternas "pued[a]n ser inadecuadas o han demostrado serlo" (Naciones Unidas, 2015, p. 29), de lo cual se desprende que no es obligatorio implementar ni agotar estas medidas. 
De esta cita, se enfatiza en el hecho de que para mantener la paz se utilizan medios bélicos aéreos, navales o terrestres. En otras palabras, lo establecido por el Capitulo VI: Arreglo pacifico de controversias y el artículo 41, tiene como excepción lo indicado en este artículo 42. Así mismo, el artículo 43 establece la obligación de los Estados Miembros de cooperar en la implementación de estos medios bélicos.

Con fundamento en el artículo 42 se puede establecer una "guerra legitimada" entendida como la autorizada por el Consejo de Seguridad con apego al mantenimiento o restauración de la paz. Resulta evidente como desde este artículo se presenta una tensión normativa con el artículo 1 de la Carta de las Naciones Unidas; pues las naciones emplearían fuerzas armadas contra un tercero para eliminar una posible amenaza.

Debido a la potencial peligrosidad de esta norma y sus alcances, preocupa que el Consejo implemente válidamente, desde un punto de vista positivista, acciones militares; aunque estas no sean idoneas. Al respecto, se debe hacer la siguiente precisión:

[N]o es suficiente que la acción militar se ajuste a la legalidad. Es imprescindible que la participación militar en una operación de mantenimiento de la paz, de imposición o de consolidación de la paz se produzca en medio del más amplio consenso posible (Doménech Omedas, 2012, p. 444).

En virtud de lo anterior, cabe resaltar que tanto las Naciones Unidas como la doctrina internacional ha buscado implementar criterios accesorios para la determinación de usos de fuerzas militares. De forma lacónica, "[1]os cinco criterios básicos de legitimidad se indican a continuación: a) Gravedad de la amenaza. [...] b) Propósito correcto. [...] c) Último recurso. [...] d) Proporcionalidad de los medios. [...] e) Balance de las consecuencias" (Doménech Omedas, 2012, p. 445), a lo enumerado anteriormente el autor agrega el "criterio de complementariedad, sostenibilidad y coordinación” (Doménech Omedas, 2012, p. 445).

Es importante añadir que un ataque armado no sería lo único a considerar como una amenaza contra la paz, dados los avances tecnológicos y la proliferación de factores de riesgo. Debido a esto, el "Secretario General de las Naciones Unidas, Kofi Annan, en el discurso que pronunció en la Asamblea General en septiembre de 2003, advirtió a los Estados Miembros que las Naciones Unidas habían llegado a una encrucijada en el camino" (Naciones Unidas, 2004, p. 3); pues resultaba necesario replantearse los peligros y las medidas que harían frente a tales amenazas. 
En función de lo anterior, se estipulan los criterios supra indicados y se establecen como principales peligros latentes para la paz las "Guerras entre estados; violencia dentro del Estado, [...] guerras civiles, abusos en gran escala de los derechos humanos y genocidio; Pobreza, enfermedades infecciosas y degradación del medio ambiente; Armas nucleares, radiológicas, químicas y biológicas; Terrorismo; y Delincuencia transnacional organizada" (Naciones Unidas, 2004, p. 4). Es de especial atención para el ensayo que, en 2003, casi a 60 años de La Carta, el principal y primer problema estipulado sea el de la guerra entre los Estados.

En ese sentido, el ordenamiento jurídico internacional prevé la utilización de la guerra por la paz, en otras palabras, se establecería una "guerra legitimada" o autorizada en el sentido que existe una norma internacional que la valida. Esta idea de la "guerra legítima" puede ser entendida como:

[E]manación directa del ordenamiento jurídico mundial: "es un principio fundamental del derecho internacional general que la guerra es permitida únicamente como una reacción contra una ilicitud sufrida - es decir como una sanción- y que cualquier guerra que no tenga este carácter es un entuerto, es decir una violación del derecho internacional (Campione, 2009, p. 145).

Por lo tanto, desde la Carta de la Naciones Unidas no se establece la eliminación de los conflictos armados, sino solo del uso "ilegítimo" de esta. A su vez, se refiere al principio de bellum justum o de guerra justa, que será entendido en el presente ensayo como "guerra legitimada", pues el fundamento resulta más acorde al sistema iuspositivista, en el cual la justicia no es el criterio utilizado, sino una norma que autoriza el actuar. Por ello, el tema de lo justo sería ajeno a este análisis. A su vez, el concepto de "guerra justa" al seguir criterios metajurídicos, es más acorde a épocas pasadas cuando predominaban sistemas de corte valorativo y axiológico más que jurídico.

Ahora bien, en la actualidad se ha retomado el término de "guerra justa" como accesorio a la guerra legítima, pues "una guerra es justa si es posible individuar [sic] una justificación moral y/o ética y/o teológica independiente y predominante respecto de lo que el derecho internacional disponga" (Campione, 2009, p.148).

Por último, se presenta la "tensión nunca resuelta y quizá irresoluble entre los dos polos de atracción en la historia de la doctrina de la 'guerra justa', el jurídico-formal y el ético-sustancial” (Campione, 2009, p.149), en la cual “[1]a primera noción identificaría una categoría jurídica, mientras que la segunda determinaría una categoría metajurídica. [...] la que es posible registrar en Kelsen sería la primera, es decir, la que remite a una dimensión jurídica” (Campione, 2009, p. 148). 
Ante este debate teórico, se podría ofrecer una respuesta desde la aplicación propiamente dicha del Capítulo VII; por tanto, en un principio, los términos utilizados son formalistas normativistas, con la salvedad de que se han ampliado los criterios de interpretación con los cuales se abordará la implementación de estos artículos. Por ello, dicho principio consiste precisamente en ese abandono de "los criterios puramente morales para establecer quién tiene razón y quién no, y debemos sustituir los juicios morales por las reglas jurídicas" (Campione, 2009, pp. 149-150); sin embargo, siempre se emplean criterios metajurídicos, lo cual responde al cambio de paradigma jurídico-filosófico del iuspositivismo por el realismo jurídico o un iusnaturalismo que emplea el derecho positivo.

De acuerdo con el artículo 43 de La Carta se establece como obligación de los Estados facilitar la implementación de las medidas ordenadas por el Consejo de Seguridad, ya sea la utilización de sus fuerzas armadas, su territorio o las demás que se consideren pertinentes, esto con el fin de asegurar que los mecanismos indicados logren su objetivo de proteger la paz.

Las medidas que se tomarán, su fundamento, así como su ejecución dependerán del Consejo de Seguridad de las Naciones Unidas, y, a su vez, del Comité de Estado Mayor.

Por último, el artículo 45 establece la forma de actuar en los estados de urgencia, e indica que "sus Miembros mantendrán contingentes de fuerzas aéreas nacionales inmediatamente disponibles para la ejecución combinada de una acción coercitiva internacional" (Naciones Unidas, 2015, p. 31); este punto es estratégico para la implementación de las medidas coercitivas.

Ante esto se debe indicar que el ser humano ha normado la guerra y así las Naciones Unidas lo han propiciado en busca de un conflicto más acorde con "los riesgos permitidos", lo cual viene a ser la muestra de la legitimación actual de la beligerancia. Ante este escenario, surgen los crímenes de guerra, comprendidos como las violaciones que dentro del conflicto podrían ser consideradas como "actos fuera del marco permitido de la guerra", es decir, se ha establecido un "deber ser" de la guerra y no una prohibición per se de esta por medio del derecho internacional humanitario.

\section{Zum ewiǵen Frieden de Immanuel Kant}

El texto de Zum ewigen Frieden escrito por el filósofo Immanuel Kant (1724-1804) es de gran importancia en el campo de la filosofía política, específicamente, en el de las relaciones internacionales. Este texto "introduce en la teoría del derecho una tercera dimensión, una innovación enriquecedora: junto al derecho estatal y 
al derecho internacional coloca el derecho cosmopolita" (Habermas, 1997, p. 61). A diferencia del derecho internacional público, el derecho cosmopolita no tiene como centro las relaciones externas entre los diversos Estados, sino que tiene como objetivo una relación íntegra entre múltiples Estados, con el fin de que estos se asuman como sujetos que componen un todo, y, por lo cual, deben regirse por las pautas del todo.

En otras palabras, el derecho cosmopolita busca reglamentar el actuar de los Estados creando un orden mundial superior a estos, es decir, la primera diferencia entre el derecho internacional público y el derecho cosmopolita es que el primero busca buenas relaciones diplomáticas mientras el segundo pretende una buena comunicación y coordinación entre los Estados con fines determinados, lo cual implica al primero, pero con un mayor nivel y jerarquía. Así mismo, desde la Segunda Guerra Mundial se "ha provocado un estremecimiento mundial y [se] ha exigido el tránsito desde el derecho internacional al derecho cosmopolita". (Habermas, 1997, p.71).

La paz perpetua es una característica importante del orden cosmopolita, pero es tan sólo un síntoma del mismo. [...] Mientras que el derecho internacional, como todo derecho en el estado de naturaleza, vale tan sólo transitoriamente, el derecho cosmopolita como el derecho sancionado estatalmente, acabaría definitivamente con el estado de naturaleza. Por eso para llegar hasta el orden cosmopolita, Kant se sirve continuamente de la analogía con aquella primera salida del estado de naturaleza (Habermas, 1997, p. 63).

Por otra parte, en vista de esta tensión normativa y posible antinomia indicada líneas arriba entre el Capítulo I y el Capítulo VII, resulta de interés analizar esta discordancia desde el texto político Zum ewigen Frieden de Immanuel Kant, pues en este texto se indican las pautas que debe cumplir un acuerdo que pretenda lograr la paz.

El texto kantiano responde al contexto político del autor, ya que este se publicó en 1795 año en el que se da la paz de Basilea entre Francia y Prusia. En él, Kant "esboza un orden de paz permanente entre los Estados que se presenta, así mismo, como la meta final de la historia humana. Desde esta meta final de la paz perpetua se explica también la necesidad de una constitución republicana en cada Estado" (Truyol \& Serra, 1998, p. 76).

De la cita del párrafo anterior, se pueden inferir dos puntos destacados: Kant escribe este texto dado el caso concreto y traslada inductivamente el hecho específico a 
la máxima de cómo sería un verdadero tratado de paz; de igual manera, el presente texto fue escrito en los últimos años del filósofo, por tanto es el resultado de su línea de pensamiento, pues es un texto orientado a hechos específicos.

Los puntos anteriores son importantes para la adecuada interpretación del texto, pues Kant construyó una filosofía estructurada, en la cual sus últimos textos son congruentes y específicos (por lo general aplicados a casos concretos) con los postulados dados en sus primeros escritos, como lo son las tres críticas kantianas (Kritik der reinen Vernunft, Kritik der praktischen Vernunft y Kritik der Urteilskraft). ${ }^{2}$ Así mismo, este texto origina el espacio dentro del área filosófica política para lo que luego fue el texto de Metaphysik der Sitten ${ }^{3}$ de 1797, el cual es de suma importancia en la filosofía del derecho y en el derecho en la época contemporánea, en general.

El texto Zum ewigen Frieden se divide en dos secciones: a) los artículos preliminares para la paz perpetua de los Estados y b) Modelo de los artículos definitivos para la paz perpetua. "Los primeros regulan las que Hans Saner llama "condiciones negativas de la paz": consisten, como vamos a ver, en una serie de prohibiciones que buscan crear un clima de confianza y concordia creciente entre los Estados" (Contreras Peláez, 2007, p. 61). En este sentido, la primera sección es condición necesaria para la segunda, por lo que solo cuando la Carta de las Naciones Unidas logre pasar el análisis de la primera, se procedería a analizarla desde la segunda sección.

\subsection{Modelo de los artículos preliminares para la paz perpetua de los Estados}

Esta sección presenta seis artículos fundamentales propuestos por Kant, que, según el autor, deben integrarse en cualquier tratado que tenga como fin la paz; de no ser así, el acuerdo devengaría en defectos que imposibilitarían el tratado sobre la paz. Los artículos serían los siguientes:

\subsection{1. "No debe considerarse válido ningún tratado de paz que se haya} celebrado con la reserva secreta sobre alguna causa de guerra en el futuro" (Kant, 1998, p. 5)

Este artículo, en función de la respuesta a la problemática, es el postulado principal. Dicha norma indica que si un tratado establece cláusulas de guerra y armamento, $o$ de cómo serían las represalias, no es en realidad un tratado que tenga como fin la paz, sino solo un cese al enfrentamiento, "simplemente de un mero armisticio, un aplazamiento de las hostilidades” (Kant, 1998, p. 5).

2 Respectivamente, la Crítica de la Razón Pura de 1781, la Crítica de la Razón Práctica de 1788, y la Crítica del discernimiento o de la capacidad de juzóar de 1790.

$3 \quad$ La metafísica de las costumbres de 1797. 
Entonces, si un tratado para la paz presenta las cláusulas indicadas anteriormente, ya sea en un futuro inmediato o no, las partes tendrán la posibilidad de irrespetar el fondo del tratado por un mero formalismo. En otras palabras, si se pactó que podrían iniciar las hostilidades, las partes podrán acudir a este mecanismo en el momento de desacuerdo, sin seguir las vías pacíficas, las cuales, en principio, son el fundamento teleológico de un verdadero tratado de paz (perpetua).

Dicho artículo surge del contexto del conflicto geopolítico entre Prusia y Austria, en el cual al firmar el tratado de Dresde en 1745 se pone fin a las "guerras de Silesia (1740-1742 y 1744-4175), pero del cual reconoce abiertamente Federico II que se trató de una mera pausa en el combate, durante la cual ambos países se rearmaron" (Contreras Peláez, 2007, p. 63); por lo tanto, el trato fue un mero cese a las hostilidades y no lo que este decía ser. Inductivamente y en virtud de esto, se indica que no puede haber una cláusula que prevé el uso de fuerzas armadas en el futuro en los tratados que tiene como objetivo la paz.

\subsection{2. "Ninģún Estado independiente (grande o pequeño, lo mismo da) podrá ser adquirido por otro mediante herencia, permuta, compra o donación" (Kant, 1998, p.6)}

En cuanto a este artículo se refiere a que el Estado, como sociedad de seres humanos, no puede ser considerado como un patrimonium, y por ello no puede ser tratado como tal; al cosificarlo como si fuera un patrimonio se eliminaría su condición de persona moral, la cual es resultado de las personas humanas dentro de él. Es decir, al anular la condición de persona moral del Estado, se anularía la representación que esta es de las personas dentro de este, por lo que se cosificarían ambas lo cual es contrario al carácter de fin -y no de medio, como sería si fueran tratados como cosas- que deben ser los seres humanos.

A su vez, Kant establece como personalidad jurídica (o un término precursor a este) al Estado, ya que no puede ser entendido como cosas, sino como "sujeto jurídico-moral: es "una sociedad de hombres sobre la que nadie más debe mandar ni disponer", "un tronco con sus propias raíces", "una persona moral"'. (Contreras Peláez, 2007, p. 70).

En este orden de ideas, un Estado que es cosificado después de un acuerdo no solo pierde su condición de persona, sino, además, el respeto que se le otorgó cuando se pactó el tratado de paz, lo que podría derivar en la posibilidad del inicio de hostilidades en su contra. 


\subsection{3. "Los ejércitos permanentes (miles perpetuas) deben desaparecer totalmente con el tiempo" (Kant, 1998, p.7)}

Este artículo concuerda con lo establecido en el punto 2.1.1., ya que la simple existencia de los ejércitos es para la guerra o la amenaza de esta: un Estado con ejército está preparado para la guerra. Además, "[e]l reforzamiento de los ejércitos genera una dinámica de desconfianza, amenaza y competición armamentística: los Estados vecinos se verán arrastrados también a reforzarse" (Contreras Peláez, 2007, p.87). Al mismo tiempo, Kant agrega:

[A]ñádese a esto que ser tomados a cambio de dinero para matar o ser muertos parece implicar un abuso de los hombres como meras máquinas e instrumentos en manos de otro (del Estado); este uso no se armoniza bien con el derecho de la humanidad en nuestra propia persona (Kant, 1998, p.7).

Lo anterior es importante porque, para la filosofía kantiana, el ser humano nunca puede ser entendido como un medio, sino como un fin, ya que al ser entendido como un medio, se le elimina su condición de ser persona y de Ser en términos kantianos, ergo, sería solo entendido como una herramienta o medio para el ejército y el interés de este.

\subsection{4. "No debe emitirse deuda pública en relación con los asuntos de política exterior" (Kant, 1998, p.8)}

Este artículo se refiere a las implicaciones que tiene la deuda exterior, pues otorga un empoderamiento del acreedor sobre el deudor, que podría afectar las relaciones pacíficas, ya sea porque el acreedor cobre en un momento donde el deudor no se encuentra facultado, o bien, cuando utilice el cobro como medida "sancionatoria" en un momento donde las relaciones estén en términos negativos. Para Kant este artículo es preliminar porque permite observar este tipo de relaciones como detonantes potenciales para el inicio de hostilidades.

Cabe hacer la precisión de que el filósofo no se opone al comercio propiamente, sino a este en el ámbito político de conflictos bélicos, pues el ámbito económico también sería utilizado como arma. A su vez "Kant no recha[za] el endeudamiento estatal como tal, sino sólo el encaminado a procurar medios para la guerra. El Estado puede endeudarse "con fines pacíficos": obras públicas, roturación de terrenos, etc." (Contreras Peláez, 2007, p. 93). 


\subsection{5. "Ningún Estado debe inmiscuirse por la fuerza en la constitución y gobierno de otro" (Kant, 1998, p.9)}

Este artículo no se refiere únicamente a la toma de territorios que se dan en las guerras o la época de conquista, sino también abarca las intervenciones de un Estado en otro cuando este se encuentre con luchas o conflictos internos, debido a que "la injerencia de potencias extranjeras sería una violación de los derechos de un pueblo independiente que combate una enfermedad interna" (Kant, 1998, p.9). Esto en virtud de que cuando un Estado interviene en otro, este último pierde ante su pueblo la legitimidad con que fue consagrado, ergo, se pierde la legitimidad de lo efectuado por este (el Estado en conflicto interno) como los tratados efectuados.

Este artículo se asemeja al "principio de no intervención por la fuerza en los asuntos internos de otro Estado" (Contreras Peláez, 2007, p. 95); sin embargo, este debe comprenderse en función de la Federación de Estados que Kant establecerá en los artículos definitivos, es decir, que se darán pautas de comportamiento para los Estados, en concordancia con el derecho cosmopolita.

\subsection{6. "Ningún Estado en guuerra con otro debe permitirse tales hostilidades que hagan imposible la confianza mutua en la paz futura, como el empleo en el otro Estado de asesinos (percussores), envenenadores (venefici), el} quebrantamiento de capitulaciones, la inducción a la traición (perduellio), etc." (Kant, 1998, pp. 9-10)

Este artículo se refiere a los momentos previos a la creación del tratado de paz, propiamente al hecho de que hay acciones que no deberían surgir en ningún momento, en especial en la guerra, pues esto puede producir una gran desconfianza entre las partes, lo cual generaría una imposibilidad en el acuerdo de voluntades a la hora de establecer tratativas de paz.

Se ha debatido si este artículo pretende establecer un ius in bellum, pero el autor lo que indica es que "una serie de reglas para una conducción "civilizada" de los conflictos bélicos no significa que se resigne a la guerra como realidad histórica insuperable, que renuncie a su visión de la paz perpetua como objetivo final" (Contreras Peláez, 2007, p. 118), sino que el autor reconoce la existencia de este como escenario previo a la implementación de un tratado.

\subsubsection{Otras consideraciones}

Cabe mencionar que, sobre estos artículos preliminares, el autor indicó:

Aunque todas las leyes citadas son leyes prohibitivas (leges prohibitivae) objetivamente, es decir, en la intención de los que detentan el 
poder. Hay algunas que tienen una eficacia rígida, sin consideración de las circunstancias, que obligan inmediatamente a un no hacer (leges strictae, como los números 1, 5, 6), mientras que otras (como los números $2,3,4)$, sin ser excepciones a la norma jurídica, pero tomando en cuenta las circunstancias al ser aplicadas, ampliando subjetivamente la capacidad, contienen una autorización para aplazar la ejecución de la norma sin perder de vista el fin, que permite (Kant, 1998, p.11).

En este sentido, los artículos 1, 5 y 6 son condición necesaria para que se pueda establecer un verdadero tratado de paz, mientras que los otros artículos quedan supeditados al adecuado establecimiento de estos.

\subsection{Modelo de los artículos definitivos para la paz perpetua}

Así como el filósofo establece pautas de cómo deberían ser las condiciones para la paz, también indican aquellas que se deben seguir para garantizar que esa condición de paz perdure. Estos artículos se explicarán brevemente a continuación.

\subsection{1. "La constitución civil de todo Estado debe ser republicana" (Kant, 1998, p.15)}

Las constituciones de los Estados republicanos tienen como meollo la libertad y la protección de esta; por tanto, se debe tener un cuerpo legislativo que norme y considere a los seres humanos como iguales.

\subsection{2. "El derecho de gentes debe fundarse en una federación de Estados libres"} (Kant, 1998, p.21)

Este artículo define que los Estados se pueden organizar y cooperar en sí como si fueran una sociedad, más no se pueden unir, porque si se da el Estado de Estados los pueblos de estos perderían sus características, lo cual haría que el Estado per se también pierda su esencia central.

\subsection{3. "El derecho cosmopolita debe limitarse a las condiciones de la} hospitalidad universal" (Kant, 1998, p.27)

Este artículo se refiere al deber de un Estado de tratar cordialmente al extranjero. No obstante, el Estado puede exigirle que salga de su territorio, siempre y cuando se le respeten sus derechos, pues irrespetar a un extranjero es irrespetar la representación de otro Estado, lo cual llevaría a conflictos entre ambos. 


\section{Carta de las Naciones Unidas desde los artículos establecidos en Zum ewigen Frieden}

En el siguiente apartado, y conforme el orden sistemático que debe seguir este análisis, es de especial atención retomar los artículos preliminares 1, 3 y 6. Desde el primer postulado se evidencia cómo la Carta de las Naciones Unidas no sigue esta línea propuesta por Kant, pues en los diversos capítulos se menciona a la utilización de fuerzas bélicas (terrestres, aéreas y navales) y no hay rastro de que se exteriorice su eliminación.

Igualmente, por el objetivo de la paz se establece una "reserva" que sería causal de movimientos bélicos; lo anterior derivaría en la utilización y el mantenimiento de la guerra. Por tanto, el artículo preliminar primero debe ser analizado en correlación con el artículo preliminar tercero, pues el mantenimiento de estas fuerzas bélicas deriva en una potencialidad de conflicto armado; además, si se le agrega el factor legitimador contemplado en La Carta, este devengaría no en el establecimiento de la paz, sino en una "guerra justificada" que sería aceptada en el concierto de las naciones pero sería una contradicción teleológica.

A su vez, el artículo 45 de La Carta define cómo los Estados Miembros deben aportar al establecimiento de estas medidas bélicas, lo cual implicaría que estos serían ejecutores de las órdenes del Consejo de Seguridad, entidad que estaría dando "órdenes de gobierno" (limitado a este tema en específico) en dicho escenario; por lo tanto, se ve una tensión normativa entre el artículo preliminar quinto y la Carta de las Naciones Unidas.

Los demás artículos preliminares no son de interés para el presente análisis, pues este se limita al fin del mantenimiento de la paz y los medios para lograr este fin. No obstante, se puede mencionar que el postulado segundo sí se ha aplicado, ya que un Estado reconocido por los demás surge de la unificación o fusión de varios Estados, o de la separación o división de un Estado anterior, y no de la apropiación de un Estado ante otro; claro está que este tema se puede ampliar con casos específicos de los últimos años.

Por otro lado, el postulado cuarto, al tener organizaciones como el Banco Mundial, se ha visto imposibilitado de lograrse, pues este ha respondido a presiones de diversos Estados que han utilizado las deudas como medio coercitivo sobre otros. Por último, el postulado sexto, el cual se refiere a aquellos países que se encuentren en conflictos bélicos o que no han optado por aplicar un tratado para acabar con este y que sea relativo a la paz, también ha tenido cierta aplicación, ya que los Estados han dispuesto normas de guerra que, aunque no tenían como fin la posibilidad 
de un tratado de paz a futuro, se da de forma indirecta. Lo anterior, pues mediante el Derecho Internacional Humanitario se han dispuesto límites a las hostilidades que son permitidas dentro de los conflictos armados, lo que implica que, de forma indirecta, una vez acabado el conflicto, se puedan establecer un tratado de paz entre las partes del conflicto. La forma indirecta de este cumplimiento radica en que estas normas humanitarias se centran en proteger a las personas y limitar el alcance de las agresiones dentro del marco bélico, por lo que la posibilidad de establecer un tratado de paz en el futuro es un mero efecto indirecto de esta protección humanitaria.

Por otra parte, si bien desde los postulados preliminares se puede indicar que la Carta de las Naciones Unidas no cumple con los criterios kantianos para ser un tratado para la paz perpetua, como mero ejercicio hipotético se puede analizar desde los postulados definitivos si esta cumple o no con el modelo de tratado kantiano.

En cuanto al primer postulado, esto no está previsto en la Carta de las Naciones Unidas, por lo tanto, si bien este documento se basa en un sistema democrático y republicano, hay Estados parte que solo poseen el primer sistema, mas no el segundo, y viceversa. El segundo y tercer postulado tampoco se han aplicado de acuerdo con la línea kantiana, pues existen diversos escenarios donde el voto de la mayoría de Estados no prevalece, como sucede por ejemplo en los temas de competencia del Consejo de Seguridad, aun cuando la incorporación a las Naciones Unidas ha sido escogida de forma soberana e independiente por cada Estado y estos tienen voz y voto dentro de la ONU.

Por otro lado, las Naciones Unidas sí fungirían como una federación de Estados, en el sentido de que ofrecen pautas generales para aplicar lo que podría entenderse como el derecho de gentes. En otras palabras, si se parte del axioma de que la Carta de las Naciones Unidas cumple con los postulados preliminares (lo cual, como se indicó, no es así), no cumpliría con los postulados definitivos para ser entendido como un tratado para la paz perpetua.

\subsection{Análisis de las medidas para mantener la paz desde los postulados kantianos}

Este es apartado se subdividirá en dos secciones: 1) Medidas para mantener la paz (desde la paz misma) y 2) Medidas para mantener la paz (apartándose de la paz misma).

\subsubsection{Medidas desde la paz misma}

La Carta estipula cómo medidas el diálogo, la negociación y demás formas de resolver conflictos pacíficamente (supra indicadas). Desde un punto de vista kantiano, estas 
medidas sí son congruentes con un documento que tiene como objetivo mantener la paz, pues nunca se saldría de ella y más bien procuraría resguardarla.

Desde un punto de vista hipotético, si únicamente existieran estas medidas, La Carta sí cumpliría con el primero de los artículos preliminares establecidos por Kant, puesto que no existirían normas donde se consagre las fuerzas armadas como medio hacia algo (guerra, conquista, simple forma de gasto administrativo), contrario sensu, se podría interpretar que la existencia de los ejércitos quedaría condicionada a la labor interna de los Estados, es decir, cumpliría función de policía y no de milicia. De igual manera, esto podría llevar al cumplimiento del artículo tercero, pues el ejecito per se dejaría de existir y pasaría a solo darse un sistema de policía. En este orden de ideas, el cumplimiento de estos dos artículos daría como resultado la imposibilidad de inmiscuirse por la fuerza en la constitución y el gobierno de otro (dado que las funciones de la policía, única fuerza vigente, no contempla dicho actuar), por lo cual se cumpliría el quinto artículo.

De esto se sigue que el Capítulo VI: Arreglo pacífico de controversias, al ser orientado en la protección de la paz por medios diplomáticos y pacíficos en las cuales concurra la buena convivencia de los Estados, sí se subsume a los postulados preliminares kantianos para lograr la paz perpetua.

\subsubsection{Medidas apartándose de la paz misma}

Como se observó en la sección correspondiente, las medidas desde la paz misma presentan gran diferencia ideológica con las que se apartan de la paz. Igualmente se determinó que las medidas consagradas en el Capítulo VI: Arreglo pacífico de controversias son congruentes con los postulados kantianos.

Ahora bien, el análisis de las medidas establecidas en el Capítulo VII: Acción en caso de amenazas a la paz, quebrantamientos de la paz o actos de agresión es sumamente diferente, debido a que se establece la utilización de las fuerzas armadas y de la guerra como medio para mantener y proteger la paz. En este punto se deben formular las siguientes preguntas: ¿puede haber paz con guerra o viceversa? ¿Se puede ir a la guerra por la paz? ¿No se está quebrantando la paz que se pretende proteger?

Estas medidas, a diferencia de las anteriores, sí se salen de la paz y entran drásticamente en el concepto de la guerra que la Carta de las Naciones Unidas pretendía eliminar. En otras palabras, la misma Carta tiene el objetivo de evitar conflictos armados para mantener la paz; sin embargo, este mismo documento indica y legitima la concurrencia de los conflictos armados. Por lo tanto, la Carta presenta una circularidad entre su fin y los medios para este, por cuanto se busca un extremo y se llega al otro, se busca la paz y se termina en la guerra. 
En respuesta a las dos preguntas, no puede establecerse la existencia de paz con guerra en un determinado contexto, puesto que son términos excluyentes entre sí e ir a la guerra por la paz tampoco tiene sentido, cuando se pretende mantener este último término.

El primer artículo kantiano se viola con la existencia del contenido del artículo 42 de La Carta, pues prevé cláusulas en las cuales se implementa la guerra como opción. Así mismo, con dichos artículos internacionales, se violentan los artículos tercero y quinto kantianos, por cuanto el ejército sigue existiendo -y se infiere que seguirá- así como se indica la existencia de la intervención estatal.

Por su parte, los artículos segundo y cuarto kantianos sí se respetan, pues no se indica nada en contra de estos.

Cabe resaltar, como ya se ha mencionado antes, que el ser humano ha normado la guerra, $y$, en estas normas, se ha cumplido con lo establecido por el artículo sexto kantiano. No obstante, si bien los redactores de La Carta han sido los redactores de dichos cuerpos normativos deontológicos bélicos, esto no se ha establecido en la Carta de la Naciones Unidas, por lo cual, con una visión delimitada al tema en concreto, se puede decir que este sexto artículo kantiano tampoco se cumple.

En síntesis, de los artículos kantianos, solo el artículo segundo y cuarto no son violentados (tampoco defendidos) por la Carta de las Naciones Unidas, mientras los artículos kantianos restantes sí están expresamente violados por dicha normativa internacional.

Por otra parte, la "guerra legitimada" no encuentra cabida en el sistema kantiano. No solo por lo indicado supra sino también desde el imperativo categórico kantiano, ya que este fundamento carecería de razón, pues el mismo no admitiría excepciones y de hacerlo no sería una verdadera norma moral, lo cual llevaría a que los Estados siempre estén con la amenaza de una posible guerra. A su vez, esta guerra al no admitir excepción, es decir, al no admitir legitimación, sería siempre inválida y no debería emplearse. En caso de utilizarse, esto conduciría a una transgresión a los artículos kantianos para la paz perpetua y establecería desconfianza entre los Estados, ergo, debilitaría el tratado y el objetivo buscado entre estos.

De forma análoga a esta imposibilidad de concebir una guerra como legitima, se puede analizar Über ein vermeinte Recht aus Menschenliebe zu lügen, el cual indica que no se pueden violentar normas morales para conseguir un fin contrario a este. En dicho texto se analiza la imposibilidad de mentir, pues al aplicar la fórmula del imperativo categórico kantiano la mentira no puede ser entendida como norma moral, ya que no habría un progreso para la humanidad. En el mismo análisis incurre la utilización de la guerra por la paz. 


\section{Conclusiones}

La Carta de las Naciones Unidas presenta, desde los postulados kantianos, una antinomia normativa inconciliable en el sentido del objetivo y medios para lograrlo, pues busca la paz, pero no por medios pacíficos, sino por medios que derivan en una "guerra legitimada". Este fenómeno de medios y el telos deriva en una incongruencia, dado que no habría una paz real. Desde el texto Zum ewigen Frieden cabe indicar que la Carta de las Naciones Unidas, en cuanto al tema de la paz, no es un tratado para la paz, sino solo un cese temporal al conflicto, ya que no cumple con los postulados preliminares del modelo kantiano, así como tampoco cumpliría con los definitivos en caso de subsanar esta falencia. Es decir, la Carta de las Naciones Unidas, no termina siendo un tratado para la paz perpetua, en tanto no busca la eliminación de la guerra y la implementación de la paz, sino que implementó causales de justificación para la guerra, las cuales ocasionan la constante amenaza y ejecución de conflictos armados y debilitan cada vez más la concreción del cumplimiento de su objetivo, lo cual conlleva una ineficacia tanto real como fáctica de las Naciones Unidas en el establecimiento de la paz.

En este sentido, resulta necesario comprender que las Naciones Unidas no podrán lograr su objetivo de paz, el cual según Kant es tautológico, ergo innecesario precisar como perpetua, pues la paz es un concepto permanente, es decir, el concepto de paz contempla la perpetuidad dentro de sus características y en caso contrario, que no sea perpetua, significa que sería un mero cese al conflicto y no el establecimiento de la paz. Lo anterior debido a que mientras se esté en la posibilidad de contar con el ejército, el cual, su mera existencia, da como alternativa la utilización bélica como respuesta o medio para lograr un fin, pone en peligro la perpetuidad de la paz, derivando en que solo sea un cese al conflicto.

Aunado a lo anterior, cabe afirmar que no existiría una implementación integral de los medios diplomáticos y pacíficos, mientas la opción de "imponer y luchar por la paz" por medio de la "guerra legitimada" esté entre las posibilidades para defender la paz. A su vez, y, en respuesta al problema planteado en la etapa introductoria, sí se cumple la hipótesis, pues las Naciones Unidas no han podido dar cumplimiento a su objetivo primario, debido a que desde su Carta constitutiva se crearon normas antagónicas con el concepto de la paz, lo que conlleva a una obstaculización formal y material; esto se ha denotado en el estudio fenomenológico de los casos en concreto.

En otras palabras, si bien las Naciones Unidas tiene una función de disminuir uno de los mayores riesgos que corre la supervivencia de la humanidad, el cual corresponde al peligro de la guerra, este no se ha podido lograr ni se logrará desde 
los postulados kantianos, debido a los puntos expuestos. A su vez, las consecuencias fácticas de esta antinomia se evidencian en que desde 1945 se han dado múltiples conflictos armados en el mundo.

A su vez, esta antinomia y lejanía con un verdadero tratado sobre la paz perpetua, ha implicado una frustración internacional, pues este fracaso en el mantenimiento de la paz y la prevención de la guerra se ha traducido en la incapacidad de la comunidad internacional de lograr el fin primario de las Naciones Unidas. Esta incapacidad fue "la base del anuncio que hizo Kofi Annan cuando asumió el cargo de Secretario General en 1997 y declaró que quería transformar la mentalidad de la ONU de una cultura de reacción a una cultura de prevención” (Sanz Caballero, 2008, p. 60).

Por otra parte, figuras como la negoción preventiva resultan idóneas con los artículos preliminares para el establecimiento de un tratado para la paz perpetua, pues esta establece un ambiente de confianza entre los Estados y plantea diversos mecanismos para evitar conflictos, y, en caso de darse, responder a ellos con medios pacíficos.

En ese sentido, las Naciones Unidas en función de cómo abordar su fin del mantenimiento de la paz debe optar por dos vías: la primera, es aceptar la imposibilidad de lograr la paz (y mucho menos la de mantenerla), ya que concurre una imposibilidad tanto formal como material. Ante este escenario, puede aumentar la atención a la diplomacia, la negociación preventiva y el ámbito de incidencia de la Comisión de Desarme de las Naciones Unidas, ya no en busca de mantener la paz, sino de prolongar el "cese del conflicto" el mayor lapso posible y con la mayor amplitud de Estados.

A su vez, estos cambios de prioridades pueden ir enfocados en crear un ambiente político ideal para la creación de un nuevo documento concerniente a la Paz Perpetua, el cual rectifique y concluya con las falencias, las carencias y las limitantes de la Carta de las Naciones Unidas.

Por otra parte, la segunda vía podría consistir en una reforma directa a dicho documento en virtud de los problemas mencionados con fundamento en el artículo 108 de La Carta. No obstante, debido a la dificultad de consenso inmediato que implicaría una reforma de esta magnitud, es que la primera vía parece más viable al ser gradual, y, en el mejor de los casos, logra que no sea solo un mero cese al conflicto, sino que sea perpetúa conforme a los postulados kantianos.

\section{Referencias}

Campione, R. (2009). El nomos de la guerra; Genealogía de la "guerra justa". Valencia, España: Tirant Lo Blanch. 
Contreras Peláez, F. J. (2007). Kant y la guerra: Una revisión de La paz perpetua desde las preguntas actuales. Valencia, España: Tirant Lo Blanch.

Doménech Omedas, J. L. (2012). La acción militar en las operaciones de paz. En J. Cardona Llorens, J. Pueyo Losa, J.L Rodríguez-Villasante y Pietro, J. L., J.M. Sobrino Heredia y M. J. Aznar Gómez M. J. (eds.), Estudios de derecho internacional y derecho europeo en homenaje al profesor Manuel Pérez González; Tomo I. (pp. 441 - 489). Valencia, España: Tirant Lo Blanch.

Habermas, J. (1997). La idea kantiana de paz perpetua: Desde la distancia histórica de doscientos años. Revista Isegoría, (16), pp. 61-90.

Kant, I. (1998). Sobre la paz perpetua (sexta edición) Madrid, España: Editorial Tecnos.

Naciones Unidas. (2004). Resumen: Un mundo más seguro: la responsabilidad que compartimos. Informe del Grupo de Alto Nivel sobre las amenazas, los desafíos y el cambio. Disponible en http://www.un.org/es/events/pastevents/a more_secure_world/pdf/brochure_sp.pdf

Naciones Unidas. (2015). Carta de las Naciones Unidas (Edición de 2015). San Francisco, Estados Unidos de América: Publicaciones de las Naciones Unidas.

Sánchez Patrón, J. M. (2013). La existencia de una controversia internacional en la Jurisprudencia del Tribunal Internacional de Justicia. En E.M. Vázquez Gómez, M.D. Adam Muñoz, y N. Cornago Prieto. (coords.). El Arreglo Pacífico de Controversias Internacionales (pp. 129 - 136). Valencia, España: Tirant Lo Blanch.

Santa Cruz, H. (1995). La creación de las Naciones Unidas y de la CEPAL. Revista de la CEPAL, (57), 17-32.

Santa María, P. A. (2013). El arreglo arbitral de las controversias internacionales: aspectos recientes. En E.M. Vázquez Gómez, M.D. Adam Muñoz, y N. Cornago Prieto. (coords.), El Arreglo Pacífico de Controversias Internacionales (pp. 129 - 136). Valencia, España: Tirant Lo Blanch.

Sanz Caballero, S. (2008). Las Naciones Unidas y la diplomacia preventiva: ¿adaptación o reforma? En J. Cardona Llorens, J. (ed.), La ONU y el mantenimiento de la paz en el Siglo XXI; Entre la adaptación y la reforma de la Carta (pp. 59 - 84). Valencia, España: Tirant Lo Blanch.

Truyol y Serra, A. (1998). Sobre la paz perpetua (sexta edición). En I. Kant, Sobre la paz perpetua (sexta edición.) Madrid, España: Editorial Tecnos. 